\title{
Real Time Monitoring of Human Body Vital Signs using Bluetooth and WLAN
}

\author{
Najeed Ahmed Khan \\ Computer Science \& IT Department \\ NED University of Engineering \& Technology \\ Karachi, Pakistan \\ M. Ajmal Sawand \\ Department of Computer Science \\ Institute of Business Administration \\ Sukkur, Pakistan
}

\author{
Marium Hai \\ Electronic Engineering Department \\ NED University of Engineering \& Technology \\ Karachi, Pakistan \\ Arwa Khuzema, Mehak Tariq \\ Electronic Engineering Department \\ NED University of Engineering \& Technology \\ Karachi, Pakistan
}

\begin{abstract}
The technology of telemedicine is emerging and advancing day by day, it is capable of taking the field of healthcare to a whole new level of personalization. A person can keep a close check on his/her health's critical signs and can receive suitable feedback if required,- to help maintain the best of health status with the help of Wireless Body Area Network (WBAN) concept. The sensor nodes can wirelessly communicate with any smart phone through an Android application to continuously monitor and have complete access to the medical data of the patient. Moreover it also aims to maintain an efficient electronic medical record of the person. Moreover, the consultant and the caretaker of the patient can have this important information remotely through an internet connection and provide with significant advice which encapsulates the term smart first aid technology.

In the proposed framework miniaturized sensors are worn on the body and non-intrusively monitor a person's physiological state. The body vital signs (e.g.: heart rate, temperature etc.) are recorded through the sensor nodes and transmit to the smart phone via Bluetooth, where the data of vital signs is stored and will further transmitted to remote locations if needed.
\end{abstract}

Keywords-Telemedicine; smartphone; Vital signs; remote locations; photoplethysmography; e-health; medical telemetry

\section{INTRODUCTION}

The global cause for death are cardiovascular disease which are increasing day by day, The death statistics are 17.3 million deaths per year and if this is the situation it is expected to increase to reach to 23.6 million by the year 2030 [1]. For improving and avoiding the death risks a contribution to the solution is made. That is an easy access, comfortable and regardless of the hardware sensors should be small, noninvasive method. The work on "WBAN" (wireless body area network) is attempted which supports mobility, data encryption, and authentication. Significant advances in science and technology such as basic developments emerging in the fields of micro/nanotechnology, wireless communication, information technology, and biomedical sciences during the past $10-15$ years has brought a clear transformation in the field of medical telemetry [2]. Medical telemetry introduces a new level of personalization, and helps an individual to have a healthy status of living. For maintaining and arranging high efficient connectivity and processing large data set technology is continuously evolving [3]. This paper is targeted to achieve a practical and affordable health care by allowing an individual (cardiac patients, athletes, sportsmen) to keep a regular check on his cardiac functioning and in any uncertain conditions ; the persons (caretakers, doctors, sports instructors) can provide instant help or advice to be safe from any major health issues.

Selection of sensor nodes is the challenging part due to the accuracy and compatibility issues. For measuring the heart rate (beats per minute) of a person, technique of photoplethysmography is used. The technique of photoplethysmography uses Infra-red LEDs (light emitting diodes) and photo detector to measure the blood volume. The sensor should be placed in those areas of body where the blood is having a higher concentration (e.g. finger tips, ear lobes etc.)[4]. Fig. 1 shows the principle of measuring heart rate of a person using technique of photoplethysmography.

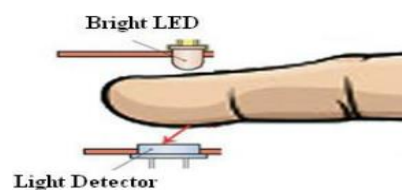

Fig. 1. Principle of photoplethysmography [5]. Contain an infrared Light detector and IR LED, used to record pulse rate

The detection of temperature can be done in two ways either through skin or through mouth. The sensor is used to detect the temperature through skin as it is the more precise way of measuring temperature in medical sciences. If the body temperature is detected to low than a normal threshold value, it is the sign of illness or if the temperature reaches to $104 \mathrm{~F}$, it is the threshold point to check body temperature variation.

\section{LiteratURE REVIEW}

In present days the world is endorsing an increasing pressure on quality and quantity of healthcare due to the increase of aging population, chronic diseases, and health consciousness of people [6]. People put more attention in 
prevention and early risk detection. The proposed model is built with the aim of providing early detection of body vital signs based on the concept of Wireless Body Area Network (WBAN). In recent years large amount of work on WBAN has been done in different areas of world but they have certain short comings.

The concept of WBAN was first presented by T. G. Zimmerman in an article from 1996, he gave these body networks the name wireless personal area network (WPAN) from the beginning [7]. Later on PAN (personal area network) was transformed in to WBAN (wireless body area network) by the accumulation of wireless data transmission devices [8] (Bluetooth, ZigBee etc.).

The increasing and continuous demand of sufficient resources placed on the medical society, increasing costs of inpatient care, and a deficit of out-patient surveillance was thoroughly described in a research paper by the scholars of the University of Twente [9].

The author of this paper has defined "extra-BAN communication" (EBAN) as communication between a BAN and other networks- IEEE 802 has established a Task Group called IEEE 802.15.6 in November, 2007 for the standardization of BAN [10]. The purpose of the group was to establish a communication standard optimized for low power, high reliability application for BANs.

In 2009, Young Dong Lee [11] designed a system for cardio vascular patients which measures electrocardiogram (ECG) and acceleration signals. He used smart shirt that can be worn easily and transmit signals using MSBN (mobile body sensor network). The shirt mainly consists of sensors for continuous monitoring the health data and conductive fabrics to get the body signal as electrodes.

There are important limitations observed for wider acceptance of the systems build up till now such as: cumbersome wires between sensors and a processing unit, lack of system integration of individual sensors, interference on a wireless communication channel shared by multiple device and unavailability of proper support for organizing huge data [12]. An interesting solution of Mobi-Health, a BSN (body sensor network) with EBAN (Ethernet body area network) connectivity to a $2.5 / 3 \mathrm{G}$ networks to provide outpatient monitoring of patients vital signs has been reported recently as well [13].

During the last few years there has been a significant increase in the number of various wearable health monitoring devices [14], ranging from simple pulse monitors, activity monitors and portable Holter monitors ${ }^{1}$ to sophisticated and expensive implantable sensors. Traditionally, personal medical monitoring systems [10], such as Holter monitors, have been used only to collect data. Systems with multiple sensors for physical rehabilitation often feature unwieldy wires between the sensors and the monitoring system [15]. These wires may limit the patient's activity and level of comfort and thus negatively influence the measured results. Recent technology advances in integration and miniaturization of physical sensors, embedded microcontrollers and radio interfaces on a single chip; wireless networking; and micro-fabrication have enabled a new generation of wireless sensor networks suitable for many applications [12].

The idea of WBAN consisting of inexpensive, light weight and miniaturized sensors can provide long-term, unobtrusive health monitoring with continuous feedback to the user about the current health status and real-time or near real-time updates of the patient's medical records.

WBAN promises a revolution in medical research through data mining of all gathered information. The large amount of collected physiological data will allow quantitative analysis of various conditions and patterns [16]. Researchers will be able to quantify the contribution of each parameter to a given condition and explore synergy between different parameters, if an adequate number of patients is studied in this manner [12].

In this paper we used the basic WBAN architecture, noninvasive techniques of heart rate and temperature detection. The paper also details the hardware and software platforms used for the monitoring of body vital signs.

\section{MATERIAL AND METHODS}

\section{A. Objective of the proposed work}

The key objective of the proposed model is to provide cardiac patients with such electronic device which can continuously monitor their heart functionality and keep medical $\log$ history that is timely updated.

In case of any alarming situations notifications in the form of messages and emails will be generated and sent to relative to take care of the patient. Along with relatives, concerned doctors will also be notified with patient's condition so that instant help can be provided. Another main focus is to provide ambulance service by sending position of the patient to ambulance station using Global Positioning System (GPS) in case of emergency.

- Wireless Body Area Network (WBAN) implemented in the proposed model consists of the following steps: Combination of sensors for detecting physiological parameters.

- The second level encompasses Android based Smart phone device which is the bus station and holds the key position in the proposed model.

- The third level and the final step of the proposed model is a wireless network (caregivers, relatives, doctors) which aims to provide feedback and related first aid services remotely.

Each level is a complex sub-system in itself requiring equal attention for assuring efficiency. Fig. 2 shows the block diagram of the proposed architecture of the model and flow of data transfer.

\footnotetext{
${ }^{1}$ http://www.polarusa.com
} 


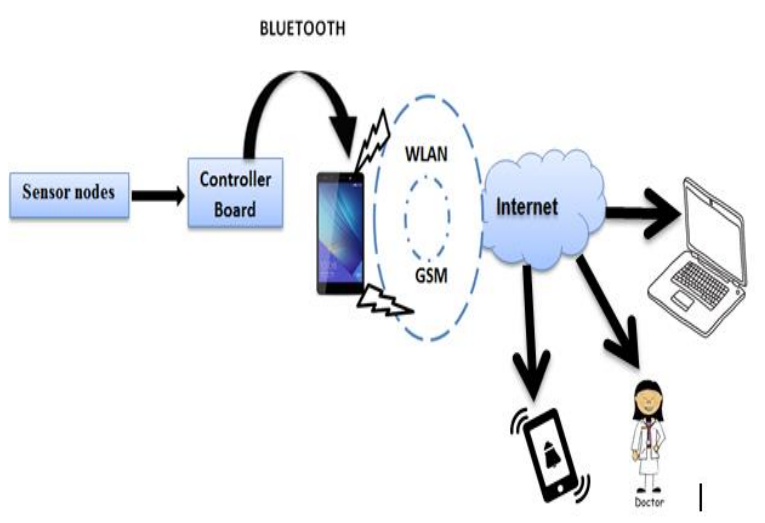

Fig. 2. System architecture of the proposed approach consists of sensors and the micro controller along with the smart phone

The proposed architecture of the model is a composite of hardware part and the software part. The hardware part consists of sensors (Plus rate sensor SEN-11574 \& Temperature sensor LM-35) and a micro controller (Arduino UNO) along with the smart phone (android device), whereas the programming for controller and an android app contribute towards the software aspect of the project. Fig. 3 shows the proposed methodology of the proposed model.

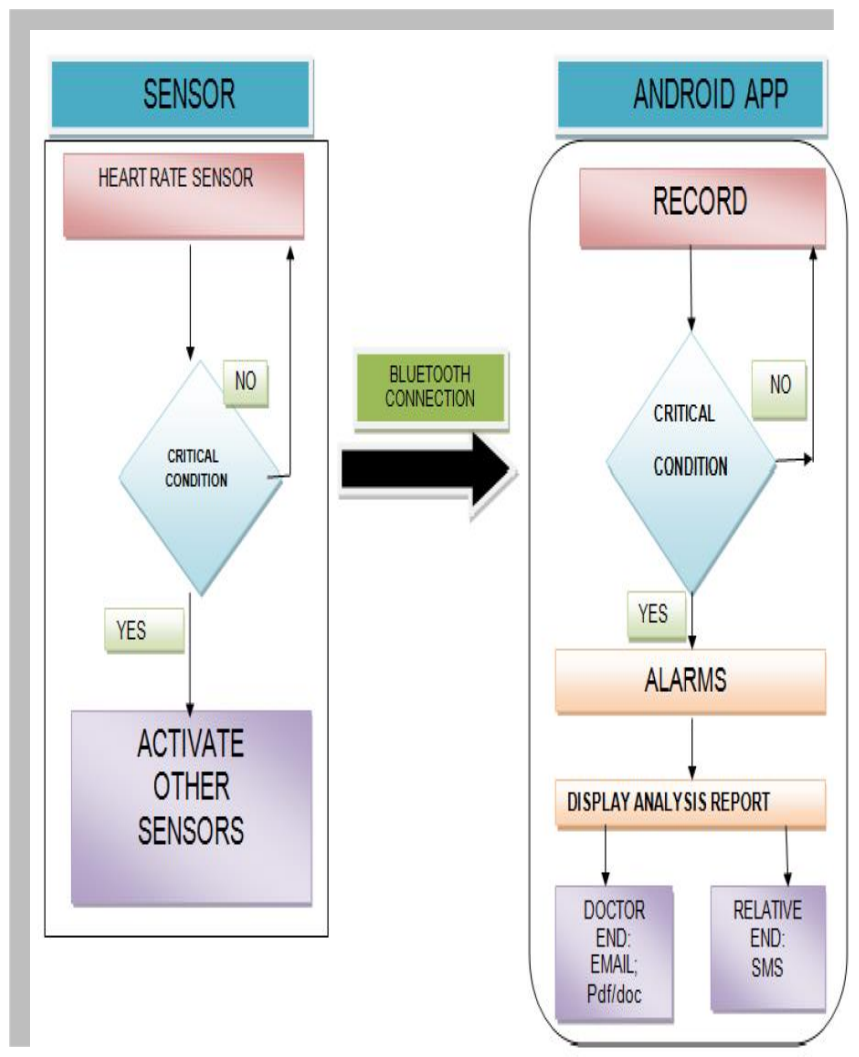

Fig. 3. Work flow of the proposed approach

\section{B. Proposed technique for the detection of pulse rate and temperature \\ PULSE RATE DETECTION}

Photoplethysmography is a technique used nowadays for monitoring patient's pulse rate. The sensor used for this task is pulse sensor (SEN-11574) which is very small in size and can be worn or wrapped around the index finger or on ear lobe. This sensor module consists of light source photo detector and infra-red LEDs (Light emitting diodes). The idea behind the concept is the optical detection of changes in the blood volume. Changes in light intensity are used to check the changes in volume level of blood which is very helpful in cardio vascular systems. Output voltages from this sensor module during the cardiac cycles will be converted into beats per minute through microcontroller board (Arduino UNO) [4].

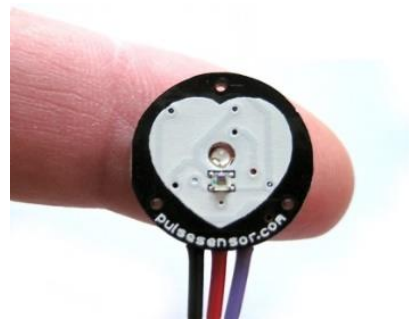

Fig. 4. Pulse rate sensor SEN-11574

\section{TEMPERATURE DETECTION}

Integrated circuit (IC) LM35 is used as a temperature measuring device. The IC is able to provide analog voltages that are proportional to temperature in Celsius $\left({ }^{\circ} \mathbf{C}\right.$.). The analog voltages are converted into digital values (temperature readings) through a microcontroller platform (Arduino UNO). LM35 is a three pin IC in which first and last pins are for supply and ground respectively whereas the middle one is for output. The following mathematical equation is used to convert output voltage of LM35 (temperature sensor) into temperature in ${ }^{\circ} \mathrm{C}$ (Celsius scale).

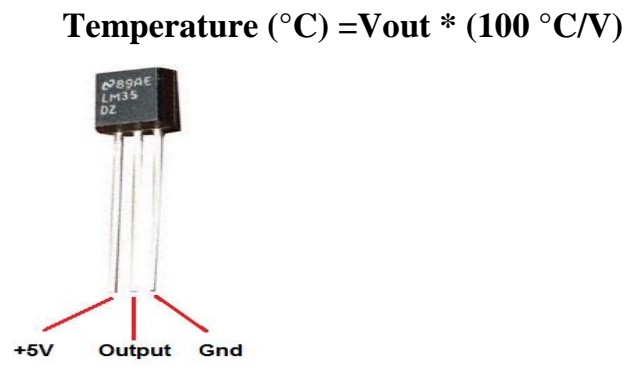

Fig. 5. Temperature sensor LM-35

\section{DATA PROCESSING}

Arduino UNO is a microcontroller board which is used here to read analog data from all the sensors and convert that data into digital form using mathematical algorithms. The 
micro-controller board will also control the series of sensors and sequence of their functioning; in a way that data from heart rate sensor is continuously read by the controller board and when it identifies any abnormal value of heart rate based on predefined threshold values, it will start reading data from other sensors too and transfer that data into the smart phone application through Bluetooth chip which is connected with this board.

\section{DATA TRANSMISSION}

Reliable communication is utmost important for health monitoring systems. Bluetooth technology is used for the transmission of data from controller board to bus station (smart phone). HC-05 module is an easy to use Bluetooth SPP (Serial Port Protocol) module, designed for transparent wireless serial connection setup. The module is compatible with micro-controller board (Arduino UNO) and can easily transfer data at the baud rate of 57600 [17]. Fig. 6 shows the connection between micro-controller board and Bluetooth. Smart android device in this project act as a personal server for the patient which collects the data of sensor nodes from Bluetooth and transmits to remote locations via internet or GSM services and also receives the feedback. This application is a significant portion of proposed model.

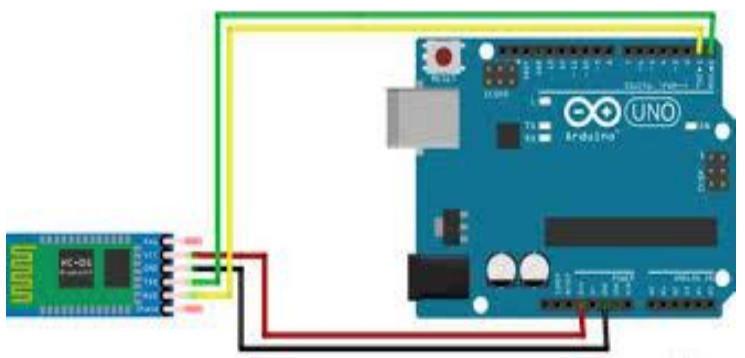

Fig. 6. The circuit diagram shows connectivity between Arduino Board and Bluetooth module

\section{DESIGNING AND DEVELOPMENT OF SOFTWARE APPLICATION}

We have chosen Android studio IDE (version 1.0) for application developing platform which was launched by Google in December 2014. It is specifically designed for android development based on the JetBrains and IntelliJ IDEA software and is a successor to Eclipse Android Development Tools (ADT) as Google's primary IDE for android application development [18].

The development of the proposed application on android involves the following steps:

- Creating the Project files

- Creating the GUI (.XML) \& JAVA files

- Bluetooth connectivity

- Running the application on virtual machine

- Running the application on actual android device [18].

GRAPHICAL USER INTERFACE (GUI) OF APPLICATION
Every screen that we see on an android smart-phone is called an activity. A unique name is given to every activity present in an android application called as activity name. Each activity comprises of two files i.e. Layout file (.XML) and the JAVA file (.java). Application in the proposed model comprises of several different activities. Fig. 7 shows the layout of some of the designed activities.

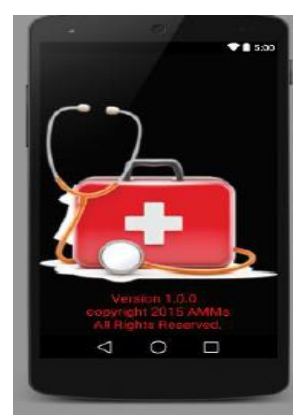

(a)

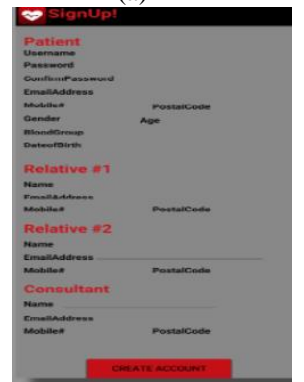

(c)

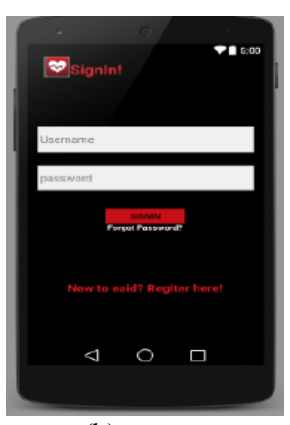

(b)

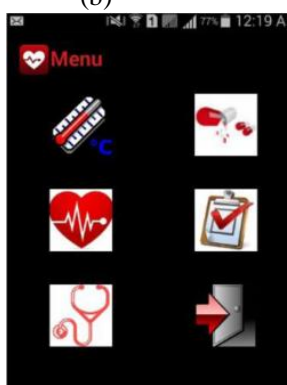

(d)
Fig. 7. Screen shots of some activities of Android Application. (a) shows the front end display of application which rests for 5 seconds before the app starts. (b) Shows the signIn activity of application. User must need to provide user Id and password to get access to the application in order to preserve privacy of the patient.(c) shows the registration form for creating account. (d) shows the menu screen of application through which the user can access the available features of application

\section{INTENTS}

Intents are basically the messaging objects used for requesting an action from other app contents i.e. calling an activity from other activity. It is communication link between different contents of an application. Fig. 8 shows the methodology followed by intents to call an activity from another one.

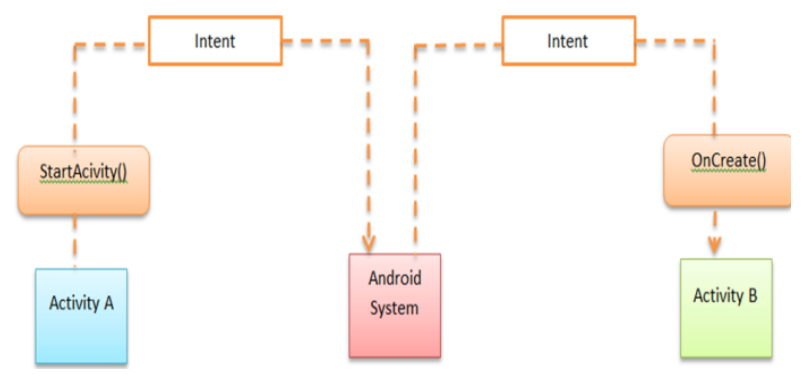

Fig. 8. Intents Methodology - responsible for the messaging objects used for requesting an action from other app contents 


\section{IMPLEMENTATION AND TESTING}

Optical technologies are well suited for non-invasive monitoring of skin blood pulsation. Radiation of the red to near infrared spectral region penetrates several millimeter under the skin surface. Skin blood pumping and transport dynamics can be monitored at different body location (e.g. fingertip, earlobe, and forehead) with relatively simple and convenient PPG contact probes. Reflection PPG method uses the back scattered Optical signals for analysis of skin blood volume pulsation [13]. In the transmission method, an optical signal change according to its absorption at the pulsation as oxygenated blood allows red wavelength more and deoxygenated blood allows infrared wavelength. It employs the principle that oxygenated blood is bright red whereas reduced or deoxygenated blood is dark red so combination of red and near infrared LEDs and photo sensors can be used to monitor the color of blood [13].The output signal of SEN11574 is an analog fluctuation in voltage as shown in fig. 9. The depiction of the pulse wave is called as Photoplethysmogram (PPG). It is the function of light intensity. When the heart pumps blood through the body; with every beat there is a pulse that travels along all arteries. A rapid upward rise in signal value occurs as the pulse wave passes under the sensor, then the signal falls back down towards the normal point [19].

1) SYSTOLE: It is the contraction period of the heart in which the heart pumps blood into all parts of the body through arteries.

2) DIASTOLE: It is the relaxation period of the heart in which heart collects blood from all body parts through veins.

The systolic peaks shown in fig. 9 are counted for the period of 60 seconds to compute the number of beats happened in one minute.

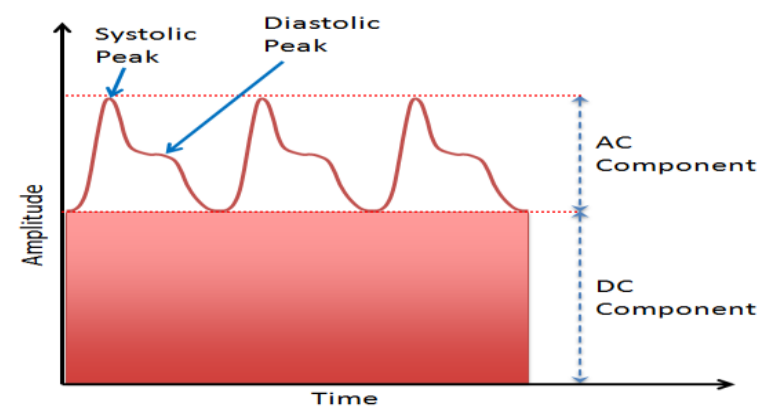

Fig. 9. Model of PPG (photoplethysmography) waveform [21]

The output wave form of SEN-11574 (pulse sensor) is recorded through oscilloscope. Fig. 10 shows the images of PPG (photoplethysmography) waveforms of two patients.

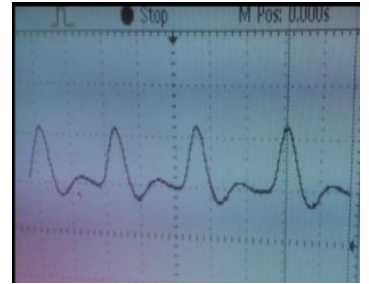

(a)

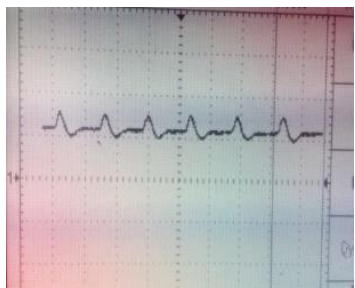

(b)
Fig. 10. Photoplethysmography output wave form of SEN-11574 sensor recorded for the patient 1 and patient 2 respectively in figure (a) and (b)

Interfacing of sensors with android application via Bluetooth is carried out by connecting micro-controller board (Arduino UNO). Bluetooth module and smart phone in proper order.5 volts supply is needed to switch on the microcontroller board. Batteries operating at 5 volts can be used for this purpose. Once the circuit is on, it starts monitoring the data of patient's vital signs and displaying it on the mobile application by transferring it through Bluetooth module. Fig. $10 \mathrm{a}$ and $\mathrm{b}$ shows the interfacing of sensor nodes and mobile application.

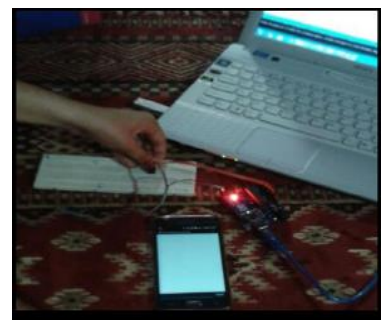

(a)

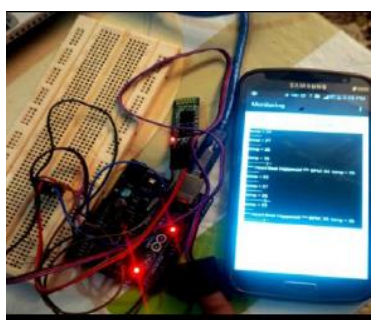

(b)
Fig. 11. Interfacing of sensor nodes with android application

Application after displaying the data extracted from sensor nodes; stores it and send it to remote locations via internet and GSM (global system for mobile communication) services. It also generates alert messages to the registered consultants and caretakers in case of any alarming situation. It also aims to send location of a patient to ambulance stations using GPS (global positioning system) services so that ambulance can reach the patient as soon as possible in order to provide first aid services to the patient.

The proposed project is simple and reliable for measuring body vital signs and aims to bring vigorous and rapid change in the field of medical telemetry. 
TABLE I. STATISTICS OF RECORDED DATA FOR DIFFERENT PEOPLE THROUGH THE DEVELOPED MODEL USING PULSE SENSOR (SEN-11574) AND BY THE AUTOMATIC MACHINE AVAILABLE FOR BLOOD PRESSURE AND HEART RATE MEASUREMENTS. THE DATA RECORDED THROUGH THE AUTOMATIC MACHINE CONSIDERED AS GROUND TRUTH

\begin{tabular}{|c|c|c|c|c|}
\hline PATIENTS & AGE & GENDER & BEATS PEI & MINUTES \\
\hline Name & Years & $\begin{array}{l}\text { Male / } \\
\text { Female }\end{array}$ & $\begin{array}{l}\text { Proposed } \\
\text { model Pulse } \\
\text { Sensor(SEN } \\
-11574)\end{array}$ & $\begin{array}{l}\text { Ground } \\
\text { Truth } \\
\text { (Omron } \\
\text { M10-IT } \\
\text { Upper Arm } \\
\text { BP and } \\
\text { heart-rate } \\
\text { Monitor }\end{array}$ \\
\hline Person 1 & 22 & Female & 92 & 90 \\
\hline Person 2 & 22 & Female & 84 & 86 \\
\hline Person 3 & 55 & Male & 73 & 72 \\
\hline Person 4 & 35 & Male & 67 & 65 \\
\hline Person 5 & 16 & Female & 82 & 85 \\
\hline Person 6 & 19 & Female & 85 & 82 \\
\hline Person 7 & 22 & Female & 82 & 82 \\
\hline Person 8 & 20 & Male & 78 & 80 \\
\hline Person 9 & 22 & Male & 82 & 82.5 \\
\hline Person 10 & 40 & Female & 74 & 75 \\
\hline Person 11 & 63 & Male & 90 & 92 \\
\hline Person 12 & 8 & Male & 82 & 85 \\
\hline
\end{tabular}

Table 1 shows the recorded data (beats per minute) of 12 different people taken from the developed model and by the automatic machine available for blood pressure and heart rate measurements. The statistics of these numerical values is graphical shows in fig. 12. Graph in fig. 12 is drawn to check the fidelity. The graph shows that the readings obtained from the test model (Red color) are in correlation with the readings obtained from an automatic blood pressure and heart rate measuring device (Blue color).

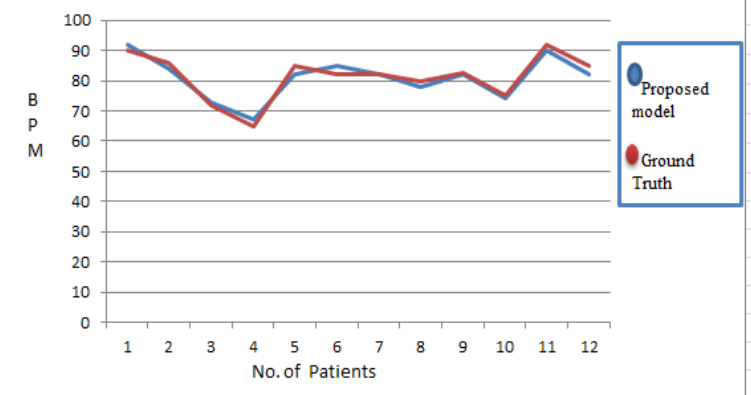

Fig. 12.Graph showing corrlation between the readings of test model and Omron M10-IT Upper Arm BP and heart-rate Monitor (ground truth)

\section{CONTRIBUTIONS}

The proposed model contributes towards the telemedicine health domain by raising the standards of health care and patient monitoring. It has a great potential in providing the society with cost effective alternative to rehabilitation of health care. It also helps the military personnel, sports persons (athletes, swimmers etc.) in keeping timely record of their health conditions. It aims to reduce sudden death risk and time saving environment to doctors, caretakers and patients as well by getting timely information about the patients and also able to provide timely suggestion to them.

\section{CONCLUSION}

Wireless Body Area Network (WBAN) proves to be a milestone in the field of wearable health monitoring providing with the services of cost effective, unobtrusive and continuous monitoring of an individual during his daily activities. As the key objective is to reduce the sudden death risks which are caused by abnormalities in vital signs through ambulatory monitoring, the proposed model has potential to offer a wide range of benefits to patients' medical personnel, and society through continuous monitoring in the ambulatory setting. It aims to provide a great benefit to patients, physicians and society by wirelessly transmitting data of vital signs of patients to remote locations so that they will be provided with first aid and medication at the earliest in case of emergency situations. By recording the daily readings of patient's heart activity and temperature variations, it helps in keeping track of every major and minor details of patient's health. The proposed model of WBAN has a great potential in providing the society with reliable and secured solutions of health care and medical problems.

In future WBAN designs can be improved for providing miniaturization, human friendly devices with more power and cost effectiveness. To advance this technology more a greater 
attention is required on portability and affordability of the sensors. To achieve ubiquitous health monitoring challenges of system design, personalization, power sources and application -specific protocols need to be resolved.

\section{REFERENCES}

[1] Subcommittee, A.S.C.a.S.S., Heart disease and stroke statistics., 2014 December.

[2] Sima Ajami , F.T., Features and application of wearable biosensors in medical care. Journal of Research in Medical Sciences, December 2015: p. $1208-1215$.

[3] Toshiyo Tamura, Y.M., Masaki Sekine, Masaki Yoshida, Wearable Photoplethysmographic Sensors-Past and Present. Electronics 3, 2014 April. Vol. 3(2): p. 282.

[4] Keith-Hynes, P.M., Benton; Robert, Antoine, A Smartphone-Based System for Real-Time Control of Blood Glucose. Electronics 3, 2014 November. no 4.

[5] M.K.Chaithanya , K.V.K.K., Avireni Srinivasulu, Blood Pressure Measurement and Data Storing Using Volume Compensation Method. IEEE, 2014 September.

[6] Abderrahim BOUROUIS, M.F., Abdelhamid UBIQUITOUS MOBILE HEALTH MONITORING SYSTEM FOR ELDERLY (UMHMSE ). International Journal of Computer Science \& Information Technology 2011 June. Vol 3, No 3.

[7] Zimmerman, T.G., Personal Area Networks: Near-field intrabody communication. IBM Systems Journal, 1996. Volume 35(Issue 3-4): p. $609-617$

[8] Javed Ahmad , F.Z., Review of Body Area Network Technology \& Wireless Medical Monitoring International Journal of Information and Communication Technology Research 2012 Feburary. Volume 2 No. 2.

[9] Aart van Halteren, R.B., Katarzyna Wac, Nicolai Dokovsky, George
Koprinkov, Ing Widya, Dimitri Konstantas, Val Jones, Rainer Herzog, Wireless Body Area Networks for Healthcare : the MobiHealth Project. Wearable eHealth Systems for Personalised Health Management, 2004. Vol. 108: p. 181-193.

[10] Milenković, A., C. Otto, and E. Jovanov, Wireless sensor networks for personal health monitoring: Issues and an implementation. Computer communications, 2006. 29(13): p. 2521-2533.

[11] Young-Dong Lee, W.-Y.C., Wireless Sensor Network Based Wearable Smart Shirt for Ubiquitous Health and Activity Monitoring. Sensors and Actuators B Chemical 2009 July. 140(2): p. 390-395.

[12] Konstantas D, H.R. Continuous monitoring of vital constants for mobile users. in 25th lntemational Conference of the IEEE EMBS September 17-21,2003 Cancun, Mexico. .

[13] Ullah, K.S.K.a.S.U.a.N. An Overview of \{IEEE\} 802.15.6 Standard. in 3rd International Symposium on Applied Sciences in Biomedical and Communication Technologies. July 2010. IEEE.

[14] Javier Andreu-Perez, D.R.L., H.M.D. Ip, and Guang-Zhong Yang, From Wearable Sensors to Smart Implants -Towards Pervasive and Personalised Healthcare IEEE, 2014.

[15] Aleksandar Milenković, C.O., Emil Jovanov, Wireless Sensor Networks for Personal health Monitoring: Issues and an implementation. Computer Communications, 2006. 29: p. 2521-2533.

[16] Syed Furqan Qadri, S.A.A., Muhammad Amjad, Masood Anwar, Suneel Shehzad, Applications, Challenges, Security Of Wireless Body Area Networks (Wbans) Sci.Int.(Lahore), 2013. 25(4): p. 697-702.

[17] Currey, M., Arduino with HC-05 (ZS-040) Bluetooth module - AT MODE. Mostly Arduino stuff, 2014 October.

[18] Lee, W.-M., Beginning Android 4 Application Development. March 2012: Wrox Programmer to Programmer.

[19] Sukhraj Kaur, D.J.M., Survey on Empirical Channel Models for WBAN International Journal of Future Generation Communication and Networking 2015. Vol. 8 , No. 2. 